\title{
The legal and ethical implication of evidence-based clinical guidelines for clinicians
}

\section{Paul Batchelor}

\author{
Senior Clinical Research Fellow, Guy's, King's and St Thomas', London, UK
}

The question that dental practitioners should be asking is whether their practice differs from published guidelines and, if so, is the deviation from the guideline justifiable.

\section{Introduction}

The quality agenda for health care delivery can be divided into three main operational sections. First, the development of standards. This will be the role of the National Institute for Clinical Excellence (NICE) and identified through the National Service Frameworks. Secondly, the delivery of the standards through clinical governance, which will involve continuing professional development and, thirdly, the monitoring of the system through three arrangements; the Commission for Health Improvement $(\mathrm{CHI})$, the National Performance Framework and the National Survey of Patient and User Experience.

As health care providers, dental practitioners will need to demonstrate that they are adopting quality assurance mechanisms. A key feature of these mechanisms will be to ensure that they are meeting defined standards and taking note of the appropriate clinical guidelines. The proliferation of the term 'guideline', along with others such as 'protocol' or 'directive', coinciding with well-publicised legal cases, may have raised uncertainty and trepidation in the mind of practitioners. In particular, the impact of such guidelines on independent clinical judgement and its relation to negligence raise unjustifiable doubts. Indeed, one of the problems facing practitioners can be guidelines that may appear to conflict. Those based on current policy at the time may not match those based solely on evidence. One example from the medical field were the guidelines issued to Health Authorities on prescribing Viagra.

This article highlights legal and ethical issues surrounding guidelines to help inform and hopefully reassure practitioners in their provision of care and, in particular, to provide a framework to reduce the possibility of litigation.

Legal issues associated with the use of guidelines

The legal basis for clinical guidelines centres on whether they are professionally accepted and well-established practice or statutory laws. The majority of current guidelines are the former and have no force of statute. One major exception concerns the provision of invitro fertilisation treatment. Under the Human Fertilisation and Embryology Act 1990, the Code of Practice restricts the number of fertilised eggs to be placed in a woman's uterus to three. Failure to comply with the Code of
Practice may lead to a penalty being imposed. However, most guidelines represent professionally accepted practice. How then do the courts assess whether the standard of practice in any given individual case was appropriate? In UK law the 'test' applied centres on the case of Bolam versus Friern Hospital Management Committee (1957; the Bolam test). In essence, it is a peergroup test by which a practitioner is judged according to what a responsible body of medical opinion skilled in that particular field of health care would have considered reasonable practice:

"a doctor is not guilty of negligence if he has acted in accordance with a practice accepted as proper by a responsible body of men skilled in that particular art".

Variation in standards can exist. Those applied to specialists may be different to those applied to generalists ${ }^{1}$. Recently, the House of Lords refined the Bolam test in the case of Bolitho versus City and Hackney Health Authority (1997), in which it was held that a judge may, on certain rare occasions, choose between two bodies of medical opinion, if one is to be regarded as 'logically indefensible'. Medical opinion must be based on logic and a measured consideration of all relevant factors. For example, in Sutton versus Population Family Planning Ltd (1981), a nurse failed to follow a referral protocol and was subsequently found to 
be negligent. But in Vernon versus Bloomsbury Health Authority, a patient was given a dose of antibiotics higher than that recommended on either the supplied drug data sheet or the British National Formulary. In this latter case, the judge preferred evidence provided by the expert witness who it was accepted had 'considerable practical experience'. The existence of a guideline does not mean that compliance is necessary, and non-compliance does not necessarily equate to negligence. For an individual to be found negligent, the person bringing the case must demonstrate that the defendant owed the patient a duty of care, that the defendant breached this duty of care by failing to provide the required standard of care, and that this caused the patient harm ${ }^{1}$.

However, this does not detract from the fact that, when providing care, a wise practitioner will endeavour to place himself in a position to be able to defend any action taken. This is where guidelines may afford the practitioner protection if they were adhered to. For guidelines to serve a useful purpose they must be monitored and updated in line with current practice, developments in treatment, or amendments that arise following complications that have been encountered and reported. In a recent study, Nicklin and Batchelor ${ }^{2}$ found that over a third of care providers failed to keep any records of complications. Without these data, the guidelines on which practitioners rely will remain unchanged and the evolving process of improving clinical practice will become static. A log of all adverse outcomes is a prerequisite for risk management and is fundamental to ensuring the quality of future patient care.

\section{Ethical implications}

In the introduction to the criteria for appraisal for national guideline use (http://www.show.scot.nhs.uk/sign/ critmain.htm\#3.2), the Scottish Intercollegiate Guidelines Network (SIGN) state:

'The purpose of clinical guidelines is to improve the effectiveness and efficiency of clinical care through the identification of good clinical practice and desired clinical outcomes.'

The ethical basis for guidelines thus has a wider implication than simply legal compliance; 'doing the right thing' goes beyond the law. It requires knowledge about laws, rules, and policies. For example, the policy of NHS Executive sees cost effectiveness as a central tenant of clinical effectiveness ${ }^{3}$. Yet, as Galbraith ${ }^{4}$ comments:

'the relation of the modern corporation to the people it comprises - their chance for dignity, individuality and full development of personality - may be at least as important as its efficiency. These may be worth having even at a higher cost of production. Why should life be intolerable to make things of small urgency?'

The answer to Galbraith's question entails value judgements. As such, clinical guidelines will not form a simple dichotomous decision methodology for care provision. A clinical guideline is not a rule that must be complied with. Ethical issues, which entail judgemental values, will influence the decision-making process. Evidencebased health care is one part of the process for ensuring high-quality care delivery. As Woolf et al ${ }^{5}$ state:

'Clinical guidelines make sense when practitioners are unclear about appropriate practice and when scientific evidence can provide an answer'.
Following from the previous point, perhaps the most positive aspect that the use of guidelines helps address is to provide an explanation as to why practitioners decide to intervene, when and how they do. In doing so, the importance of the role of the practitioner will become clearly and publicly defined. That can only be beneficial to both the public and the profession.

In summary, the fact that a guideline exists does not mean that the dental practitioner has a duty to follow the recommendations specified in it. However, dental practitioners should be aware of the content of published guidelines, especially those that are evidence-based, produced using the rigours of Cochrane methodologies. The question that dental practitioners should be asking is whether their practice differs from published guidelines and, if so, is the deviation from the guideline justifiable. This has both ethical and moral connotations in addition to the legal aspects. Most importantly, to answer the question, all practitioners should have in place risk management procedures.

1. Hurwitz B. Legal and political considerations of clinical practice guidelines.Br Med J 1999; 318:661-664.

2. Nicklin P, Batchelor PA. Current understanding of clinical governance: a study of dental care providers. Br Dent J (In press).

3. NHS Executive. Promoting Clinical Effectiveness: a Framework for Action in and through the NHS. Leeds: Department of Health, 1996.

4. Galbraith JK. The Affluent Society 4th ed. London: Penguin, 1991.

5. Woolf SH, Grol R, Hutchinson A, Eccles $M$, Grimshaw J. Potential benefits, limitations, and harms of clinical guidelines. Br Med J 1999; 318:527-530. 